\title{
VALORACIÓN DE LA ADOPCIÓN DE EMBRIONES HUMANOS CONGELADOS DESDE EL PUNTO DE VISTA DE LA FILOSOFÍA MORAL, LA ÉTICA LAICA Y DOS RELIGIONES MONOTEÍSTAS
}

\author{
Justo Aznar ${ }^{1}$, Miriam Martínez ${ }^{2}$ Pedro Navarro² \\ Resumen: La búsqueda de la eficacia en la fecundación in vitro hace que se produzcan más embriones que los que se implantarán, \\ lo que produce un excedente de embriones, que es congelado. Esto hace que ineludiblemente el número de embriones \\ humanos congelados aumente. Entre las soluciones para dichos embriones humanos congelados está la donación/adopción \\ de los mismos. Ineludiblemente esta práctica conlleva objetivos problemas éticos. En este trabajo se evalúa la eticidad de la \\ donación/adopción de embriones humanos congelados desde la perspectiva de la filosofía moral, lo que podríamos llamar \\ una "ética laica" y dos de las religiones monoteístas: la musulmana y la judía.
}

Palabras clave: embriones humanos congelados, adopción de embriones humanos congelados, valoración ética, valoración moral

Valuating frozen human embryo adoption from a moral philosophical point of view, lay ethics and two monotheist religions

\begin{abstract}
The search for IVF efficacy leads to a higher embryo production than it is necessary for implantation; this results in an excess of embryos which are kept frozen. This amount of frozen embryos inevitably increases. The donation/adoption are among the possible solutions for these frozen embryos. However, this practice has objective ethical problems. This article considers the ethical aspects of the donation/adoption of frozen human embryos from the point of view of moral philosophy,
\end{abstract} from what we could call "secular ethics" and from two monotheistic religions: Muslim and Jewish.

Key words: frozen human embryos, frozen human embryo adoption, ethical considerations, moral considerations

Valoração da adoçáo de embrióes humanos congelados do ponto de vista da filosofia moral, a ética laica e duas religióes monoteístas

Resumo: A busca da eficácia na fecundação in vitro faz com que se produzam mais embrióes dos que se implantarão, o que produz um excedente de embrióes, que é congelado. Isto faz com que inquestionavelmente o número de embrióes humanos congelados aumente. Entre as soluçóes para os ditos embrióes humanos congelados está na doaçáo/adoçãoo dos mesmos. Ineludivelmente esta prática implica objetivos problemas éticos. Neste trabalho se avalia a eticidade da doação/adoção de embriôes humanos congelados a partir da perspectiva da filosofia moral, o que poderíamos chamar uma "ética laica" e duas religiôes monoteistas: a mulçumana e a judia.

Palavras-chave: embrióes humanos congelados, adoção de embriôes humanos congelados, valoração ética, valoração moral

\footnotetext{
${ }^{1}$ Instituto de Ciencias de la Vida, Universidad Católica de Valencia, España Correspondencia: justo.aznar@ucv.es

${ }^{2}$ Facultad de Enfermería, Universidad Católica de Valencia, España
} 


\section{Aspectos generales}

Como es sabido la eficacia de la fecundación in vitro es baja, pues se sitúa alrededor del 30\%(1). Por otro lado, es sabido que la eficacia de esta técnica y consecuentemente el índice de embarazos conseguidos y nińos nacidos vivos es la principal razón para el reclutamiento de clientes en las clínicas que ofrecen la reproducción asistida. Para conseguir sus objetivos, son varias las medidas que se promueven por dichas clínicas, pero posiblemente la más eficaz es disponer de un elevado número de embriones congelados de la pareja que desea conseguir el hijo, para así poder repetir, en sucesivas sesiones, la implantación de alguno de ellos y aumentar así las posibilidades de una gestación.

Para conseguir un número suficiente de embriones humanos congelados en cada ciclo de fecundación in vitro se fecundan varios de ellos, por lo general entre 10 y 12. En la primera ocasión se implantan entre dos y tres embriones, dejando el resto congelado. Si este primer intento falla, se descongelan otros dos o tres para ser implantados, y así sucesivamente hasta generalmente un máximo de cinco intentos. Si tras ello no se consigue el deseado embarazo generalmente se da por fallido el proceso.

Esta práctica inevitablemente condiciona que el número de embriones humanos que se almacenan congelados aumente progresivamente. Como consecuencia, se plantea un importante debate ético acerca de qué hacer con los embriones humanos congelados sobrantes. Existen cuatro soluciones principales: a) dejarlos congelados indefinidamente; b) dedicarlos a experimentaciones biomédicas; c) descongelarlos y dejarlos morir, y d) darlos en adopción(1). A esta última posibilidad vamos a referirnos en este trabajo.

Los aspectos técnicos de la adopción de embriones humanos congelados los hemos evaluado en una publicación anterior(1). En esta ocasión vamos a referirnos a la evaluación ética de esta práctica desde tres vertientes: a) la filosofía moral; b) lo que podríamos denominar una ética laica, y c) el juicio que esta práctica merece en las religiones monoteístas. Con respecto a estas últimas valoraremos aquí lo que afecta a las religiones musulma- na y judía, para abordar en un próximo artículo todo lo que concierne a las religiones cristianas, y muy especialmente a la católica.

\section{La adopción de embriones humanos congelados a la luz de la filosofía moral}

Son pocos los trabajos recientes que abordan la licitud o ilicitud moral de la adopción de embriones humanos congelados a la luz de la filosofía moral; sin embargo, ello es abordado, a nuestro juicio, magníficamente por Adriano Pessina en un reciente trabajo(2).

En relación con ello, Pessina, manifiesta que, desde un punto de vista antropológico, "se trata de evaluar, aunque solo sea desde una vertiente argumentativa, si la adopción prenatal se puede proponer como éticamente aceptable y en qué condiciones". Estima que se deben tener en consideración varios aspectos:

"El problema de la licitud moral de la adopción prenatal es, por sí mismo, distinto del problema de la suerte que pueden correr los embriones congelados y no puede presentarse como una solución práctica para resolverlo".

"Hay que preguntarse si la adopción prenatal es la única solución ética aceptable, aunque con ella solo se pudiera alcanzar un objetivo teórico, pero no práctico, del problema de los embriones congelados".

"No se puede ignorar que la adopción de los embriones congelados va a favor indirectamente de aquellos que defienden la crioconservación embrionaria, lo que podría abrir la puerta a la cesión de embriones y así favorecer de alguna forma la procreación heteróloga”.

Pero, a mi juicio, la aportación más interesante de Pessina es que "se pueden plantear dos líneas argumentativas al evaluar la adopción de embriones humanos congelados. En la primera, se asume que la vida humana es un valor absoluto, inconmensurable, y como tal no equiparable a ningún otro. En la segunda, se admite que la vida humana es un valor básico, porque es una condición necesaria para sustentar otros bienes humanos, pero no suficiente para alcanzar los fines específicos del 
hombre, lo que comporta que el valor de la vida humana puede ser pospuesto a otros valores, por ejemplo al dar la vida por otro".

Si se admite el primer principio, "no habría objeción para la adopción prenatal, incluso esta se podría presentar como moralmente positiva y no solamente lícita”. Si se admite la segunda opción argumentativa, "la vida del embrión humano se debería defender únicamente por medios proporcionados, ordinarios y moralmente legítimos, siendo en este sentido la única posibilidad la de invitar a la madre biológica a que le implanten el embrión congelado de su hijo y a llevar el embarazo a término. Otras opciones podrían ser consideradas desproporcionadas y extraordinarias, lo que podría llevar a conculcar otros valores fundamentales relacionados con la dignidad de la persona humana y de la procreación humana”.

En relación con todo ello, Pessina manifiesta: "personalmente pienso que la segunda posibilidad es la que mejor expresa la concepción moral personalista", concluyendo que "la adopción prenatal de hecho y de derecho contribuye -al margen de la búsqueda de los bienes que pueden animar a algunos - a falsear el significado ético y antropológico de la generación humana, a privar al nasciturus del derecho a nacer en y desde el vientre de su madre y en condiciones que respeten su naturaleza de hijo".

Termina Pessina afirmando que, "en la historia del hombre los conflictos morales requieren una compleja reflexión y darse cuenta que no siempre aquello que parece en sí mismo como bueno lo es verdaderamente, cuando se abandonan los nexos que unen la bondad con la verdad".

Tras esa reflexión teórica, Pessina se manifiesta moralmente contrario a la adopción de embriones humanos congelados y expone(2) las razones que a su juicio justifican dicha ilicitud, al afirmar que:

- No respeta el derecho del hijo a desarrollarse en el seno de su madre y no en el útero de cualquier otra mujer.

- Tampoco respeta la unidad psicofísica del embrión, que en la fase de su desarrollo in- trauterino mantiene una estrecha relación con su madre, lo que influye en su desarrollo, tanto físico como psicológico, lo que posteriormente puede afectar de forma importante al desarrollo de su personalidad.

- Trastoca tanto el concepto de maternidad como el de genitalidad, porque legitima la escisión entre los componentes biológicos, afectivos y relacionales de la procreación, como ocurre en la procreación extracorpórea, la maternidad subrogada o incluso ante la posibilidad de la clonación humana.

- Induce a una lectura puramente "funcional" de la maternidad y avala directamente la maternidad subrogada, de la cual no se distingue si no es por la intención de custodia del neonato.

- Es contraria al concepto mismo de familia, porque induce a las parejas que pueden tener niños por la vía natural a privilegiar este tipo de adopción prenatal.

Valoración de la adopción de embriones desde el punto de vista de una ética laica

\section{Aspectos generales}

En la valoración ética de la donación/adopción de embriones congelados desde la perspectiva de una ética laica se pueden considerar distintos aspectos, especialmente: a) el bien del posible nacido; b) la dignidad de las personas en relación con su instrumentalización y comercialización; c) la justicia en cuanto a igualdad se refiere; d) la autonomía reproductiva; e) la elección de los donantes y de los receptores; f) no favorecer el comercio de embriones; g) garantizar la existencia del consentimiento libre y expreso de los donantes; h) asegurar que existen suficientes datos clínicos para un correcto uso del material donado; i) garantizar la capacidad legal, tanto de usuarios como de profesionales, y j) no utilizar embriones abandonados $(3,4)$.

Pero, con independencia de todo lo expuesto, nos parece que, en relación con la valoración ética de la donación/adopción de embriones humanos congelados, aquellas personas o instituciones que 
no tengan dificultades éticas para utilizar la procreación asistida o la congelación de embriones, no tendrán tampoco dificultades morales para la adopción de embriones humanos congelados, incluso considerarán ésta una solución positiva para dichos embriones, pues, según ellos, si los embriones no son utilizados por los progenitores con fines reproductivos, su donación/adopción es éticamente más defendible que cualquier otro destino que se pueda dar a los embriones sobrantes, ya que poner en marcha un proceso reproductivo para intentar que nazca un nińo es, a su juicio, la mejor solución para los embriones humanos congelados. Por ello, pueden pensar que, aunque la crioconservación de embriones humanos sea en sí misma éticamente negativa, la donación/ adopción es éticamente positiva, ya que lo que se persigue es, en primer lugar, contribuir a construir familias, es decir, ayudar a parejas infértiles a conseguir un hijo, y, en segundo lugar y como consecuencia directa de lo anterior, proteger un bien primario del embrión: su vida. Consecuentemente, gran parte de los expertos o instituciones laicas ven en la donación/adopción de embriones humanos congelados una alternativa para el destino de dichos embriones éticamente más valiosa que utilizarlos para investigaciones biomédicas, destruirlos o dejarlos conservados indefinidamente.

Sin embargo, se estima que el número de embriones que podría beneficiarse de la donación es tan reducido que difícilmente podría esta práctica contribuir de forma efectiva a solucionar el problema de los embriones humanos actualmente congelados, por lo que la donación/adopción de embriones se justificaría esencialmente desde un punto de vista ético, por ser un acto de generosidad por parte de las parejas donantes, que tratan de ayudar a parejas que desean conseguir un hijo y que por cualquier circunstancia tienen dificultades para ello(1).

También otros estiman que, desde un punto de vista ético, la donación/adopción de embriones podría equipararse a la adopción de niños ya nacidos, aunque en este tipo de adopción, la posnatal, solo se buscan unos padres que deseen conseguir un hijo, lo que sin duda es una acción solidaria $y$, en consecuencia, éticamente positiva; pero en la descongelación, además de proporcionar para el embrión descongelado unos padres, se incluye el hecho positivo de dar a ese embrión una oportunidad de vivir, lo que añade, según algunos, un plus de bondad ética a la descongelación y donación/adopción de embriones humanos congelados sobre la adopción posnatal.

Al margen de estos aspectos positivos de la donación/adopción de embriones, una posible dificultad desde una perspectiva ética laica es que al llevarse a cabo la descongelación se pudiera vulnerar el principio de indisponibilidad del cuerpo humano, aunque para algunos esta dificultad podría ceder éticamente ante otros valores y principios de mayor importancia, como es la preservación de la vida humana del propio embrión congelado, o ayudar a construir una familia a parejas que lo deseen y que tengan insalvables dificultades para conseguirlo.

Resumiendo, somos de la opinión que, desde una perspectiva laica, se puede afirmar que la donación/adopción de embriones congelados es éticamente aceptable por la gran mayoría de las personas e instituciones.

Ya en un orden práctico, al realizar la valoración médica de la donación/adopción de embriones humanos congelados, desde una perspectiva laica vamos a referirnos, como botón de muestra, a lo que opinan diversas instituciones públicas que representan a amplios colectivos sociales o profesionales, pues nos parece que estas tienen un valor representativo mayor.

\section{Informe del Comité Nacional de Bioética del Conse- jo de Ministros italiano}

El Comité Nacional de Bioética del Consejo de Ministros italiano emitió el 19 de noviembre de 2005 el documento "L'adozione per la nascita" (5), en el que valora éticamente la adopción de embriones congelados, destacando los valores de solidaridad, generosidad y responsabilidad que deben caracterizarla, aunque también señalando el riesgo de que mediante la adopción de embriones se pueda legitimar, aunque sea indirectamente, la procreación asistida heteróloga, haciendo además hincapié en que esta objeción no debería afectar a aquellos que piensan que la procreación asistida no presenta dificultades éticas. 
Refiriéndonos ya explícitamente al documento que estamos comentando, después de evaluar este distintos aspectos propios de la adopción de embriones humanos congelados, concluye que es éticamente aceptable proponerla para resolver, al menos en parte, el problema ético que se plantea en relación con los embriones humanos congelados sobrantes de la fecundación in vitro.

Adicionalmente, subraya determinadas condiciones que deberían ser tenidas en consideración para que dicha adopción pueda ser éticamente aceptable, entre ellas, a) que se certifique legalmente el abandono de los embriones; b) que se evite la comercialización de la técnica; c) que se garantice el derecho a la privacidad de los padres biológicos; d) que se haga constar explícitamente el consentimiento de los padres para que el embrión pueda ser donado en el futuro; e) que se informe adecuadamente a la mujer o pareja adoptantes de las condiciones éticas y legales que rigen la adopción, y f) que, en caso de que nazca un niño, este deberá ser considerado a todos los efectos jurídicos como un hijo legítimo o natural de la mujer que lo ha gestado.

Sin embargo, a dicho documento se adjuntaron lo manifestado en algunos votos de calidad, que consideran que la ley es excesivamente restrictiva, y también lo de otros miembros de dicho Comité, como Bompiani, Di Pietro y Sgreccia, que por el contrario se abstuvieron de votar, porque pensaban que el debate sobre la adopción de embriones humanos congelados no había alcanzado todavía suficiente madurez como para realizar una adecuada valoración ética sobre él, y que, por otro lado, la solución planteada por este documento, y aprobada por la Comisión Plenaria del Comité Nacional de Bioética italiano, parecía ser teórica e imperfecta, y no encuadrada en un contexto de garantía real de salvaguardia de la vida de todos los embriones congelados.

Informe del Comité Ético de la Sociedad Americana de Medicina Reproductiva (ASRM) del año 2009 (3)

Esta asociación se manifiesta favorable a la adopción de embriones humanos congelados, al expresar su bondad ética cuando dicha adopción se dirige a la construcción de familias, opinión con la que además están de acuerdo otros comités de bioética $(6,7)$. Sin embargo, también manifiesta que, por no tener los embriones humanos estatuto de persona, no parece correcto utilizar el término de adopción, que a su juicio es equívoco, porque refuerza la idea de que el embrión tiene todo el derecho para ser considerado un ser humano adulto, lo que no parece justificable, pues a los embriones humanos se les puede asignar un estatuto moral más elevado que a otros tejidos humanos, pero nunca pueden ser considerados como personas, reafirmando así lo que en un documento de 1986 de este mismo Comité se manifestaba(8): "el (pre)embrión merece más respeto que otros tejidos humanos porque potencialmente puede llegar a ser una persona, y porque tiene un significado simbólico para mucha gente, pero que no puede ser tratado como una persona porque no ha desarrollado las características de la personalidad humana y no ha alcanzado aún su potencial biológico". Igualmente, en un documento posterior(9), la ARSM vuelve a afirmar que los embriones merecen un especial respeto, pero que nunca se les puede atribuir el mismo estatus moral que a las personas.

Esta misma Sociedad puntualiza algunos aspectos que se deben tener en cuenta en la donación/ adopción de embriones humanos congelados, afirmando que(10): a) los embriones no pueden ser vendidos con ánimo de lucro; b) los embriones únicamente deben ser generados para uso de uno mismo, y c) los embriones son el producto de una ambigua unidad parental y como tal son trasferidos.

Resumiendo, se puede a nuestro juicio afirmar que la gran mayoría de los colectivos éticos públicos o entidades profesionales se muestran favorables a la adopción de embriones humanos congelados, siempre y cuando se tengan en cuenta determinados requisitos que determinen el buen hacer de estas prácticas.

Valoración moral de la adopción de embriones congelados en la religión musulmana

En general, se puede afirmar que la donación/ adopción de embriones humanos congelados muestra dificultades morales en distintas religiones(11-13). Y con referencia específica al mundo 
musulmán, el deseo de tener una propia prole es muy fuerte. El mismo Corán, libro santo de los musulmanes, manifiesta que: "La riqueza y la descendencia son dos derechos objetivos en el mundo musulmán"(14). También una oración común entre los musulmanes es: "concédenos esposas e hijos que sean el placer de nuestros ojos"(15), por lo que la infertilidad es considerada como una desgracia(16).

Por ello, a las familias infértiles se les anima a que traten de solucionar su problema de infertilidad, pero siempre dentro de los límites que establece la Shari'ah(17).

Hasta recientemente, los problemas de infertilidad eran fundamentalmente tratados, médicamente o por técnicas quirúrgicas encaminadas a solucionar problemas orgánicos. La utilización de dichos tratamientos no ofrecía especiales dificultades morales para los musulmanes, pero la introducción de las técnicas de reproducción asistida ha hecho cambiar la situación sustancialmente, pues estas tecnologías condicionan que el proceso procreativo pase de la intimidad de los esposos a medios artificiales desarrollados en un laboratorio, y en muchas circunstancias - y esto es lo más grave dentro del mundo musulmán- a que terceras personas participen en estos procesos, lo que ha hecho que su licitud moral haya cambiado radicalmente.

Según Fadel(18), las principales reglas morales que rigen el mundo musulmán se encuentran en la Shari'ah y el Corán. Aquellas situaciones que no se contemplan en estas dos fuentes son reguladas por disposiciones legales denominados "ljtihad"(19), que evalúan la gran mayoría de los problemas que pueden afectar a la conducta moral de los musulmanes, y, por supuesto, dentro de ellas, a las relaciones familiares $(15,19)$. Estas normas relativas a la familia parten del principio que cada niño tiene derecho a conocer la identidad de su padre y de su madre, circunstancia que, como es natural, afecta a las técnicas de procreación asistida e igualmente a la valoración moral de la donación/adopción de embriones humanos congelados, pues la implantación de dichos embriones condiciona que los padres biológicos sean diferentes de los adoptivos, que van a ejercer como tales durante toda la vida del hijo.
De acuerdo con lo anteriormente comentado, la religión musulmana — desde un punto de vista teórico — tiene objetivas dificultades morales para utilizar las técnicas de reproducción asistida. Sin embargo, desde un punto de vista práctico, no parece que estas dificultades afecten demasiado a la conciencia moral de la población musulmana, pues en gran parte del mundo musulmán la industria de la fecundación in vitro es floreciente. Así, en Egipto, país con 70 millones de habitantes, se han abierto más de 50 centros de procreación asistida, y en el Líbano, con 4 millones de habitantes, más de 15(20).

En relación con la adopción de embriones humanos congelados, una primera dificultad es la de establecer un único criterio moral, debido a la gran variedad de grupos religiosos existentes, aunque son dos los predominantes, Sunitas y Chiitas, por lo que nos referiremos principalmente a ellos. Otra dificultad es la existencia de diversas reglas morales dentro de estos mismos grupos, aunque la principal, y más seguida es la Sharia. Pero sobre todo, lo que diversifica ampliamente los criterios morales es la existencia de las "fatwa", reglas morales fundamentadas en la interpretación que destacados clérigos islámicos hacen de la Sharia y de otras importantes reglas morales musulmanas $(21,22)$.

Entre los sunitas, la primera y más influyente "fatwa” emitida sobre esta materia fue en 1980 (solamente dos ańos después del nacimiento de Louise Brown, la primera nińa nacida en el mundo por fecundación in vitro), por el Gran Shaykh de la Universidad de Al-Azhar, de Egipto(11,12,20), considerado como el principal foro de pensamiento sunita, grupo religioso que representa el 80 a 90\% de los musulmanes de todo el mundo.

En general, se puede afirmar que las técnicas de procreación asistida que implican donación de gametos, especialmente semen, merecen para los sunitas una catalogación moral negativa $(12,20,23)$, al considerar esta práctica incluso como un adulterio por parte de la mujer que recibe el semen que no es de su legítimo marido(15,20,24,25). Pero, además, según ellos, el uso de este tipo de semen rompe la línea genealógica familiar, lo que es muy negativamente considerado(25). Es posible decir que, siempre en general, a los musulmanes sunitas 
se les prohíbe el uso de las técnicas que implican donación de gametos por terceros y, entre ellas, la adopción de embriones humanos congelados.

Más complejas, por diversas razones, son las normas morales que rigen para los musulmanes chiitas, especialmente radicados en Irán y Líbano, aunque en general se puede afirmar que estas son más permisivas que las de los sunitas. Existe una "fatwa" del Ayatolá Khameini, sucesor de Khomeini, que se muestra más liberal para admitir como moralmente aceptables las técnicas de reproducción asistida que utilizan gametos de terceros(12). En efecto, Khamanei manifiesta, en su primera "fatwa", que la donación de ovocitos no está legalmente prohibida(20), pues es esta práctica la única opción que puede tener una mujer chiita cuyo marido sea infértil para conseguir un hijo(26). Pero de todas formas, como anteriormente se ha comentado, la multiplicidad de "fatwas" entre los chiitas hace que no exista un criterio común. Algunos, contrariamente a la opinión de Khameini, manifiestan que la donación/adopción de embriones humanos congelados rompe la paternidad, por lo que no puede ser moralmente aceptable(20).

En Irán, país de mayoría chiita, las cosas son aún más complicadas. En una conferencia celebrada en Teherán sobre Donación de gametos y embriones, se discutió ampliamente la legitimidad moral de la adopción de embriones humanos congelados, sin llegar a una conclusión unánimemente aceptada(20). Sin embargo, una reciente ley iraní especifica que las parejas que deseen adoptar embriones humanos congelados pueden dirigirse por escrito a un juez para que autorice la transferencia embrionaria(26). De todas formas, conviene subrayar que las leyes que rigen esta práctica son tan novedosas que la gran mayoría de las clínicas de procreación asistida iraníes no disponen aún de las disposiciones legales que regulan la adopción de embriones humanos congelados(26), por lo que la ambigüedad legal y moral sobre esta práctica es máxima.

En resumen, se puede afirmar que los musulmanes sunitas se muestran más reacios a considerar como moralmente aceptable la donación de gametos por terceros y la adopción de embriones humanos congelados, y que los chiitas son más propicios a aceptar moralmente estas prácticas.

Valoración moral de la adopción de embriones congelados en la religión judía

En la religión judía no es fácil encontrar textos concretos que se refieran a la moralidad de la adopción de embriones humanos congelados, sí en cambio los hay sobre la donación de embriones por terceros(12), por lo que, por analogía, se podría extrapolar dicha valoración a la de adopción de embriones humanos congelados.

En relación con ello, Klein(12) manifiesta que, aunque algunos pacientes que utilizan estas prácticas son fervientes judíos(27), la mayoría de los rabinos ortodoxos se manifiestan muestran dudosos sobre la licitud moral de la adopción de embriones humanos congelados(12). Estiman que la principal dificultad moral expresada en la ley judía sobre la donación de ovocitos es la cuestión de la identidad maternal, al plantear quién es la verdadera madre del niño nacido: la biológica o la receptora de los ovocitos y gestante del embrión, por lo que la donación de ovocitos no parece ser aceptable para la mayoría de las autoridades rabínicas tradicionales(28), y probablemente, por extensión, tampoco lo será la donación/adopción de embriones humanos congelados.

\section{Referencias}

1. Aznar J, Martínez M, Navarro P. Adopción de embriones congelados. Therapeia 2016; 8 (in press).

2. Pessina A. La cosiddetta adozione pre natale. Questione etiche. (Texto pro-manuscripto. Per concessione dell'autore)..

3. The Ethics Committee of the American Society for Reproductive Medicine. American Society for Reproductive Medicine: defining embryo donation. Fertil Steril 2009; 92: 1818-1819.

4. Marqueta Sobrino J, Arnott Fernández I, Marquetta Marques B, Torres Vives M. Límites en la donación de embriones. In Pedreña N. Donación de embriones. Salerno (Italia): Momento Médico SRL; 105-109.

5. Comitato Nazionale per la Bioetica. Ministri PdCd. L'adozione per la nascita (APN) degli embrioni crioconservati e residuali derivanti da procreazione medicalmente assistita (P.M.A). Comitato Nazionale per la Bioetica; 18-XI-2005. 
6. National Institutes of Health (NIH), Ad Hoc Group of Consultants to the Advisory Committee to the Director. Report of the Human Embryo Research Panel. Bethesda, Maryland: National Institutes of Health (NIH); 1994.

7. Department of Health, Task Force on Life and the Law. Executive summary of assisted reproductive technologies, analysis and recommendations for public policy. United States: Department of Health; 1998. Available from: https://www.health. ny.gov/regulations/task_force/reports_publications/execsum.htm

8. Ethics Committee of the American Fertility Society. The donation of preembryos. Fertil Steril 1986; 46: 295-315.

9. Ethics Committee of the American Society for Reproductive Medicine. Defining embryo donation: a committee opinion. Fertil Steril 2013; 99(7): 1846-1847.

10. Practice Committee of American Society for Reproductive Medicine; Practice Committee of Society for Assisted Reproductive Technology. Recommendations for gamete and embryo donation: a committee opinion. Fertil Steril 2013; 99(1): 47-62.

11. Klein JU, Sauer MV. Ethics in egg donation: past, present and future. Semin Reprod Med 2010; 28: 322-328.

12. Klein JU. Religious views: the impact of traditional theological opinion on the practice of third-party reproduction. In Sauer MV. Principles of oocyte and embryo donation. London: Springer-Verlag; 2013.

13. Brody B. Current religious perspectives on the new reproductive techniques. In Bartels DM, et al. Beyond Baby M. The Human Press Inc; 1990. 45-63.

14. Holy Quran 14: 46.

15. Holy Quran 25: 74.

16. Fadel HF. Assisted reproductive technologies: an islamic perspective. Islamic Med. Ass'n 1993; $14: 17$

17. Shari'ah, Islamic jurisprudence, designates the rules and regulations governing the lives of Muslims, derived principally from the Qur'an and Hadith. The Encyclopedia of Islam; 1996: 321.

18. Fadel HE. The Islamic viewpoint on new assisted reproductive technologies. Fordham Urban Law Journal 2002; 30(1): $147-157$.

19. Council on Islamic Education (cited 2002 October 10). Available from http://www.cerisnet.pitt.edu/resource/councilon-islamic-education

20. Inhorm MC. Making muslim babies: IVF and gamete donation in sunni versus shi'a islam. Culture, Medicine and Psychiatry 2006; 30: 427-450.

21. Lane SD, Rubinstein RA. The use of fatwas in the production of reproductive health policy in Egypt. In $90^{t h}$ Anual Meeting of the American Anthropological Association. Chicago; 1991.

22. Zuhur S. Of milk mothers and sacred bond: Islam, patriarchy and new reproductive technologies. Creighton Law Review 1992; 25: 1725-1738.

23. Rizvi SM. New Techniques in Human Reproduction In Rizvi SM. Marriage and Morals in Islam. Islamic Education $\&$ Information Center. (Accessed on 14 March 2014). Available from: https://www.al-islam.org/marriage-and-moralsislam-sayyid-muhammad-rizvi/chapter-five-new-techniques-human-reproduction

24. Inhorn MC. Fatwas and ARTS: IVF and gamete donation in Sunni v. Shi'a Islam. Special issue on "assisted reproductive technologies". J Gend Race Justice 2006; 9: 291-317.

25. Larijani B, et al. Ethical and religious aspects of gamete and embryo donation and legislation in Iran. $J$ Relig Health 2007; 46: 399-408.

26. Tremayne S. The moral, ethical and legal implications of egg, sperm, and embryo donation in Iran. Ann Arbor 2005 May 19.

27. Schenker J. Assisted reproductive technology: perspectives in Halakha (Jewish religious law). Reprod Biomed Online 2008; 17: 17-24.

28. Reichman E. The rabbinic conception of conception: an exercise in fertility. Tradition 1996; 31: 33-63.

Recibido: 25 de julio de 2014

Aceptado: 17 de abril de 2015 